\title{
ON SS-SUPPLEMENTED SUBGROUPS OF FINITE GROUPS AND THEIR PROPERTIES
}

\author{
XIUYUN GUO \\ Department of Mathematics, Shanghai University, \\ Shanghai 200444, P.R. China \\ e-mail:xyguo@shu.edu.cn \\ and JIAKUAN LU \\ Department of Mathematics, Guangxi Normal University, \\ Guilin 541004, Guangxi, P.R. China
}

(Received 9 November 2010; revised 31 October 2011; accepted 19 January 2012; first published online 30 March 2012)

\begin{abstract}
A subgroup $H$ of a finite group $G$ is called $S S$-supplemented in $G$ if there exists a subgroup $K$ of $G$ such that $H K=G$ and $H \cap K$ is $S$-quasinormal in $K$. In this paper, we characterize the finite groups in which every subgroup is $S S$-supplemented and the influence of $S S$-supplementation of some subgroups on the structure of finite groups is considered. Some recent results on $S S$-quasinormal subgroups and $C$-supplemented subgroups are strengthened and enriched.
\end{abstract}

2010 AMS Mathematics Subject Classification. 20D10, 20D20.

1. Introduction. All groups considered in this paper are finite.

A group $G$ is said to be factorized into its subgroups $A$ and $B$ if $G$ is the product of $A$ and $B$. Obviously, the structure of the factorized group $G=A B$ is restricted by its subgroups $A$ and $B$. There has been interest in the past in investigating the structure of the factorized group $G=A B$ by means of the structure of $A$ and $B$. For instance, in 1955, Itô found an impressive and very satisfying theorem. He proved in [17] that $G=A B$ is a metabelian group if $A$ and $B$ are abelian. The most famous theorem of this type was due to Kegel (see [18]) and Wielandt (see [28, 29]) as they stated the solvability of the factorized group $G=A B$ if $A$ and $B$ are both nilpotent. It is also well-known that the group $G=A B$ is nilpotent if $A$ and $B$ are both normal nilpotent subgroups of $G$. However, it is known that the factorized group $G=A B$ is not necessary supersolvable if both $A$ and $B$ are normal supersolvable subgroups of $G$ (see [3]). Thus, the following interesting question arises:

Let $\mathcal{F}$ be a formation (may be a saturated formation). What will be the conditions needed for the subgroups $A$ and $B$ so that the factorized group $G=A B \in \mathcal{F}$ when both $A$ and $B$ are in the formation $\mathcal{F}$ ?

In answering the above question, Asaad and Shaalan first proved a theorem in 1989 [1] by showing that if $G=H K$ is a factorized group with supersolvable subgroups $H$ and $K$ such that every subgroup of $H$ is permutable with every subgroup of $K$, then $G$ is supersolvable. In 1992, Maier in [23] further proved that the above result can also be obtained by considering the general completeness property of all saturated formations containing the class of supersolvable groups. Along this direction, Ballester-Bolinches and some others have investigated the totally permutable products and the mutually 
permutable products of finite groups, and consequently many interesting results have been given (for example, see $[4,6]$ ).

Motivated by the above results, we now call a subgroup $H$ of a group $G S S$ supplemented in $G$ if there exists a subgroup $K$ of $G$ such that $G=H K$ and $H \cap K$ is an $S$-quasinormal subgroup in $K$. In this case, the subgroup $K$ is said to be an $S S$-supplement of $H$ in $G$.

Recall that a subgroup $H$ of a group $G$ is $S$-quasinormal in $G$ if $H$ permutes with every Sylow subgroup of $G$. After the introduction of the above concept by Kegel (see [19]), the structure of a group has been extensively investigated under some additional assumptions on the subgroups of a given group (see $[\mathbf{2}, \mathbf{2 4}]$ ). On the other hand, a subgroup $H$ of a group $G$ is called a complemented subgroup of $G$ if there exists another subgroup $K$ of $G$ such that $G=H K$ and $H \cap K=1$. By using the concept of complemented subgroups, Hall established a fundamental theorem for solvable groups in [14] by proving that a group $G$ is solvable if and only if every Sylow subgroup is complemented. Recently, the authors have also investigated the finite $p$-nilpotent groups with some subgroups c-supplemented in [13]. Research on the complemented subgroups of a given group still continues and many related results have been recently obtained (see [5, 11, 12]).

In this paper, we first describe the relationship between the $S S$-supplemented subgroups and the complemented subgroups or $S$-quasinormal subgroups of a given group $G$. Next, we study the structure of the finite groups whose subgroups are $S S$ supplemented. Some applications of our results are considered so that a number of related results in the literature are extended and generalized.

2. Preliminaries. In this section, we first discuss the properties of $S S$ supplemented subgroups and give some lemmas which will be used in the sequel. For the sake of convenience, we recall that a subgroup $H$ of a group $G$ is $C$-supplemented in $G$ if there exists a subgroup $K$ of $G$ such that $G=H K$ and $H \cap K \leq H_{G}$ (see [7]), where $H_{G}$ is the core of $H$ in $G$. It is obvious that a subgroup $H$ of a group $G$ is $C$-supplemented in $G$ if and only if there exists a subgroup $K_{1}$ of $G$ such that $G=H K_{1}$ and $H \cap K_{1}=H_{G}$. Hence, the concept of $C$-supplemented subgroups can be regarded as a generalization of both $C$-normal subgroups and complemented subgroups; therefore, it is worthwhile to investigate the structure of a group by considering its $C$-supplemented subgroups. On the other hand, we recall a new concept (see [21]), which is a generalization of $S$-quasinormality. A subgroup $H$ of a group $G$ is called to be $S S$-quasinormal in $G$ if there is a subgroup $K$ of $G$ such that $G=H K$ and $H$ permutes with every Sylow subgroup of $K$. Many interesting results on $S S$-quasinormality of a group have been recently given by $\mathrm{Li}$ and others (for instance, see [21, 22]).

Definition 2.1. A subgroup $H$ of a group $G$ is said to be $S S$-supplemented in $G$ if there exists a subgroup $K$ of $G$ such that $G=H K$ and $H \cap K$ is S-quasinormal in $K$. In this case, we say that $K$ is an $S S$-supplement of $H$ in $G$.

It is clear that a $C$-supplemented subgroup of a group $G$ must be $S S$-supplemented in $G$. We now assume that $H$ is a $S S$-quasinormal subgroup of a group $G$. Then, there exists a subgroup $K$ of $G$ such that $G=H K$ and $H$ permutes with every Sylow subgroup of $K$. Let $P$ be a Sylow subgroup of $K$. Then, by $H P=P H$, we deduce that $(H \cap K) P=P(K \cap H)$. This shows that $H$ must be $S S$-supplemented in $G$. On the other hand, a $S S$-quasinormal subgroup of a group may not be $C$-supplemented and a 
$C$-supplemented subgroup of a group may not be $S S$-quasinormal (see Example 2.2). Furthermore, the following Example 2.3 illustrates that a SS-supplemented subgroup of a group may be neither C-supplemented nor SS-quasinormal. Hence the class of all $S S$-supplemented subgroups in a group contains properly both the class of all $C$ supplemented subgroups and the class of all $S S$-quasinormal subgroups in the group.

EXAMPLE 2.2. Let $G=S_{4}$ be the symmetric group of degree 4 and let $H=\langle(34)\rangle$. Then, $H$ is $C$-supplemented in $G$ since $G=H A_{4}$ and $H \cap A_{4}=1$. However, $H$ is not $S S$-quasinormal in $G$ because $H P \neq P H$ when $P=\langle(123)\rangle$.

Let $P=\left\langle x, y: x^{16}=y^{4}=1, x^{y}=x^{3}\right\rangle$. Then, $\Phi(P)=\left\langle x^{2}, y^{2}\right\rangle=\left\langle x^{2}\right\rangle \times\left\langle y^{2}\right\rangle$. It is easy to see that $H=\left\langle y^{2}\right\rangle$ is $S$-quasinormal in $P$ and so $S S$-quasinormal in $P$. However, $H$ is not $C$-supplemented in $P$.

ExAmple 2.3. Let $G$ be the direct product of $S_{4}$ and $P$ with $S_{4}$ and $P$ as in Example 2.2. Now let $H=C_{2} \times P_{1}, K=A_{4} \times P$, where $C_{2}=\langle(34)\rangle, P_{1}=\left\langle y^{2}\right\rangle$ and $A_{4}$ is the alternating group of degree 4. Then, $G=H K$ and $H \cap K$ is $S$-quasinormal in $K$ since $H \cap K \cong P_{1}$. Hence, $H$ is $S S$-supplemented in $G$. However, $H$ is neither $C$-supplemented nor $S S$-quasinormal in $G$.

We now give some basic properties of $S S$-supplemented subgroups.

LemMA 2.4. Let $G$ be a group and $H$ an $S S$-supplemented subgroup of $G$. Then, the following statements hold:

(1) If $M$ is a subgroup of $G$ and $H \leq M$, then $H$ is $S S$-supplemented in $M$.

(2) If $N$ is a normal subgroup of $G$ and $N \leq H$, then $H / N$ is $S S$-supplemented in $G / N$.

(3) Let $\pi$ be a set of primes. If $H$ is a $\pi$-subgroup of $G$ and $N$ is a normal $\pi{ }^{\prime}$-subgroup of $G$, then $H N / N$ is $S S$-supplemented in $G / N$.

(4) If $L$ is a subgroup of $G$ and $H \leq \Phi(L)$, then $H$ is $S$-quasinormal in $G$.

Proof. By the hypotheses, there exists $K \leq G$ such that $H K=G$ and $H \cap K$ is $S$-quasinormal in $K$. Let $K_{1}=M \cap K$. Then, $M=H K_{1}$ and $H \cap K_{1}=H \cap K$ is $S$ quasinormal in $K_{1}$. This shows that $H$ is $S S$-supplemented in $M$ and thus (1) is proved.

It follows from $G=H K$ that $H / N \cdot K N / N=G / N$. By using the well-known Dedekind identity, we have $H / N \cap K N / N=N(H \cap K) / N$. For any prime number $p$, it is well known that any Sylow $p$-subgroup of $K N / N$ has the form $K_{p} N / N$, where $K_{p}$ is a Sylow $p$-subgroup of $K$. Thus, $(H / N \cap K N / N)\left(K_{p} N / N\right)$ is a subgroup of $G / N$ since $(H \cap K) K_{p}$ is a subgroup of $G$. This implies that $H / N \cap K N / N$ is $S$-quasinormal in $K N / N$. Therefore, $H / N$ is $S S$-supplemented in $G / N$ and (2) is proved.

Since $(|N|,|H|)=1, N \leq K$ and $N H \cap K=N(H \cap K)$. This shows that $N H \cap K$ is $S$-quasinormal in $K$, and hence, $N H$ is $S S$-supplemented in $G$. By (2), $H N / N$ is $S S$-supplemented in $G / N$ and (3) follows.

Since $H \leq \Phi(L), L=H(L \cap K)=L \cap K$. It follows that $K=G$ and $H$ is $S$ quasinormal in $G$. Thus, (4) holds and the proof is completed.

The following lemmas are known results of $S$-quasinormal subgroups of a given group $G$.

Lemma 2.5. ([19]) Let $G$ be a group and $H \leq G$. If $H$ is $S$-quasinormal in $G$, then $H$ is subnormal in $G$.

LEMMA 2.6. ([24]) If $H$ is a p-subgroup of a group $G$ for some prime $p$, then $H$ is $S$-quasinormal in $G$ if and only if $O^{p}(G) \leq N_{G}(H)$. 
LEMMA 2.7. ([8]) If $A$ is subnormal in $G$ and $B$ is a minimal normal subgroup of $G$, then $B \leq N_{G}(A)$.

Recall that a class $\mathcal{F}$ of groups is called a formation if $G \in \mathcal{F}$ and $N \unlhd G$ then $G / N \in \mathcal{F}$, and if $G / N_{i} \in \mathcal{F}, i=1,2$, then $G / N_{1} \cap N_{2} \in \mathcal{F}$. In addition, if $G / \Phi(G) \in$ $\mathcal{F}$ implies $G \in \mathcal{F}$, then we call $\mathcal{F}$ a saturated formation. A well-known example of saturated formations is the class $\mathcal{U}$ of supersolvable groups.

Concerning saturated formations, we have the following known results.

LEMMA 2.8. ([25]) Let $\mathcal{F}$ be a saturated formation containing $\mathcal{U}$, let $G$ be a group with a normal subgroup $H$ such that $G / H \in \mathcal{F}$. If $H$ is cyclic, then $G \in \mathcal{F}$.

LEMMA 2.9. ([26]) Let $\mathcal{F}$ be a saturated formation containing $\mathcal{U}$ and $G$ a group with a solvable normal subgroup $H$ such that $G / H \in \mathcal{F}$. If for every maximal subgroup $M$ of $G$, either $F(H) \leq M$ or $F(H) \cap M$ is a maximal subgroup of $F(H)$, then $G \in \mathcal{F}$.

3. $S S$-supplemented subgroups of a group. A group $G$ is said to be $S S$ supplemented if every subgroup of $G$ is $S S$-supplemented in $G$. In this section, we first investigate the solvability of groups by using $S S$-supplemented subgroups and then the $S S$-supplemented group will hence be characterized.

THEOREM 3.1. Let $G$ be a group. Then, $G$ is solvable if and only if every Sylow subgroup of $G$ is $S S$-supplemented in $G$.

Proof. If the given group $G$ is solvable, then every Sylow subgroup of $G$ is complemented and hence $G$ is $S S$-supplemented.

Conversely, we assume that every Sylow subgroup $P$ of $G$ is $S S$-supplemented in $G$. Then, by definition, there exists $K \leq G$ such that $P K=G$ and $P \cap K$ is $S$-quasinormal in $K$. By Lemma 2.5, $P \cap K$ is subnormal in $K$. Note that since $P \cap K$ is a Sylow subgroup of $K$, we can easily see that $P \cap K$ is also a normal Sylow subgroup of $K$. By applying the Schur-Zassenhaus theorem [9, Theorem 6.2.1], we have $K=(P \cap K) K_{p^{\prime}}$, where $K_{p^{\prime}}$ is a Hall $p^{\prime}$-subgroup of $K$. Now $G=P K=P K_{p^{\prime}}$ and $P \cap K_{p^{\prime}}=1$. Hence $P$ is complemented in $G$. The theorem is proved.

By using the same arguments as in Theorem 3.1, we deduce the following corollary.

Corollary 3.2. Let $G$ be a group and $H$ a Hall subgroup of $G$. Then $H$ is complemented in $G$ if and only if $H$ is $S S$-supplemented in $G$.

If we only assume that all maximal subgroups are $S S$-supplemented in a group $G$, then $G$ need not be solvable. In fact, $L_{2}(7), L_{2}(11)$ and $L_{5}(2)$ are nonabelian simple groups in which every maximal subgroup is complemented (see [20], main theorem). However, we have the following result.

THeORem 3.3. Let $G$ be a group. Then, $G$ is solvable if and only if every maximal subgroup of $G$ has a subnormal SS-supplement in $G$.

Proof. Let $G$ be a solvable group and $H$ a maximal subgroup of $G$. We now proceed to show that $H$ has a subnormal $S S$-supplement in $G$. Assume that $H_{G} \neq 1$. Consider $G / H_{G}$. By using induction on $|G|$, we know that $H / H_{G}$ has a subnormal $S S$-supplement $K / H_{G}$ in $G / H_{G}$. Clearly, $K$ is a subnormal $S S$-supplement of $H$ in $G$. Assume that $H_{G}=1$. Let $N$ be a minimal normal subgroup of $G$. Then, $H N=G$ and $H \cap N \leq H_{G}=1$. Hence, $N$ is a normal $S S$-supplement of $H$ in $G$. 
Conversely, assume that the result is not true so that we can let $G$ be a counterexample of minimal order. Consider a maximal subgroup $H$ of $G$. Then there exists a subnormal subgroup $K$ of $G$ such that $H K=G$ and $H \cap K$ is $S$-quasinormal in $K$. If $G$ is a nonabelian simple group, then $K=G$ since $H \neq G$. By Lemma 2.5, we know that $H$ is subnormal in $G$ and hence $H=1$. It follows that $G$ is solvable, which is a contradiction. Now, we let $N$ be a minimal normal subgroup of $G$. Then, it is easy to see that the hypothesis is still true for the quotient group $G / N$. By the minimality of $G$, we infer that $G / N$ is solvable. Furthermore, we may assume that $N$ is the unique minimal normal subgroup of $G$ and $N$ is not contained in $\Phi(G)$. Then, in this case, we can let $M$ be a maximal subgroup of $G$ with $M_{G}=1$. By our hypothesis, there exists a subnormal subgroup $K$ of $G$ such that $M K=G$ and $M \cap K$ is $S$-quasinormal in $K$. Since $K$ is subnormal in $G$, Lemma 2.5 implies that $M \cap K$ is subnormal in $G$. Assume $M \cap K \neq 1$, then we may take a minimal subnormal subgroup $L$ of $G$ contained in $M \cap K$. Since $L \cap N \unlhd L$, either $L \cap N=1$ or $L \leq N$. By Lemma 2.7, $N$ normalizes $L$. If $L \cap N=1$, it follows that $N L=N \times L$ and $L \leq C_{G}(N)=1$. Suppose $L \leq N$, then $L^{G}=L^{N M}=L^{M} \leq M_{G}=1$. We also get $L=1$, a contradiction. Hence $M \cap K=1$. By using the same arguments, we can similarly prove that all minimal subnormal subgroups of $G$ are contained in $N$. Let $N=N_{1} \times \cdots \times N_{r}$, where each $N_{i}$ is isomorphic to a fixed nonabelian simple group. Then, it is easy to see that $N_{1}, \ldots, N_{r}$ coincide with all minimal subnormal subgroups of $G$. Without loss of generality, we may assume that $N_{1} \leq K$. Then, there exists a prime $p$ such that $p$ divides $|G: M|=|K|$. By applying [3, Lemma 3, P.121], we obtain that $N$ is solvable, a contradiction. The proof is now completed.

The following corollary is a direct consequence of Theorem 3.3.

COROLlary 3.4. ([19]) A group $G$ is solvable if and only if for every maximal subgroup $M$ of $G$, there exists a subnormal subgroup $K$ of $G$ such that $G=M K$ and $M \cap K \leq M_{G}$.

REMARK. From the proof it can be noted that Theorem 3.3 is also valid if 'subnormal' is replaced by 'normal'. The same is valid for Corollary 3.4.

If a group $G$ has a solvable maximal subgroup $M$ such that $M$ is $S S$-supplemented in $G$, then $G$ need not be solvable, for instance, $A_{5}$. However, we have the following result.

THEOREM 3.5. Let $G$ be a group. Then, $G$ is solvable if and only if $G$ has a solvable maximal subgroup $H$ such that $H$ has a normal SS-supplement $K$ in $G$.

Proof. If $G$ is solvable, then $G$ has a normal maximal subgroup $H$. It is easy to see that $H$ has a normal $S S$-supplement $K$ in $G$, namely $G$. Conversely, assume that the theorem is not true. Then, we let $G$ be a counterexample of minimum order. If $H_{G} \neq 1$, then $H / H_{G}$ is a solvable maximal subgroup of $G / H_{G}$ and $K H_{G} / H_{G}$ is a normal $S S$ supplement of $H / H_{G}$ in $G / H_{G}$. The choice of $G$ implies that $G / H_{G}$ is solvable and therefore $G$ is solvable, a contradiction. Hence, $H_{G}=1$. Let $N$ be a minimal normal subgroup of $G$ and $C=C_{G}(N)$. Then, it follows from [8, A, 17.2] that either $N$ is the unique minimal normal subgroup of $G$ and $C \leq N$ or $G$ has precisely two minimal normal subgroups $N$ and $R$ so that $N \simeq R$ is nonabelian, and hence, $R=C$ and $N \cap H=1=R \cap H$. By our hypotheses, we deduce that $H \cap K$ is $S$-quasinormal in $K$ and therefore, by Lemma 2.5, we know that $H \cap K$ is subnormal in $K$ and is hence in $G$. Now, assume that $H \cap K \neq 1$ and let $L$ be a minimal subnormal subgroup of $G$ contained in $H \cap K$. If $L \leq N$, then $L^{G}=L^{N H}=L^{H} \leq H_{G}=1$, a contradiction. 
This shows that $L$ is not contained in $N$ and $L$ is analogously not contained in $R$. It hence follows that $N \cap L=1=R \cap L$. On the other hand, by Lemma 2.7, we have $N L=N \times L$ and therefore $L \leq C$, which contradicts $C \leq N$ or $C=R$. Hence, we conclude that $H \cap K=1$. This implies that $G=[K] H$ and $K$ is a minimal normal subgroup of $G$.

Now, we let $T$ be a minimal normal subgroup of $H$. Then, $T$ is clearly an elementary abelian $p$-group for some $p \in \pi(H)$. Since $C_{K}(T)$ is normalized by both $H$ and $K$, we know that $C_{K}(T) \unlhd G$. If $C_{K}(T)=K$, then $T \leq H_{G}$, a contradiction. Hence, $C_{K}(T)=$ 1. It now follows from [9, Theorem 6.2.2] that $K$ is a $p^{\prime}$-group. By [9, Theorem 6.2.3], $K$ contains a unique $T$-invariant Sylow $q$-subgroup $Q$ for every prime $q \in \pi(K)$. For any $h \in H$, we have $\left(Q^{h}\right)^{T}=\left(Q^{T}\right)^{h}=Q^{h}$, that is, $Q^{h}$ is also a $T$-invariant Sylow $q$-subgroup of $K$, and thereby $Q=Q^{h}$. Consequently, we have $[Q] H=G=[K] H$ and so $K=Q$ is a $q$-group. This implies that $G$ is a solvable group, a contradiction. Thus, the proof is completed.

We now characterize the $S S$-supplemented groups.

THEOREM 3.6. Let $G$ be a group. Then, the following statements are pairwise equivalent.

(1) $G$ is an SS-supplemented group.

(2) $G$ is supersolvable, every Sylow subgroup of $G / \Phi(G)$ is elementary abelian and every subgroup of $\Phi(G)$ is $S$-quasinormal in $G$.

(3) every subgroup of $G / \Phi(G)$ is complemented and every subgroup of $\Phi(G)$ is $S$ quasinormal in $G$.

Proof. (1) $\Rightarrow(2)$. We first prove that $G$ is supersolvable. By the hypotheses and Theorem 3.1, $G$ is solvable. Let $N$ be a minimal normal subgroup of $G$. Then, $N$ is an elementary abelian $p$-group for some prime $p$. By Lemma 2.4(2), it is known that $G / N$ is $S S$-supplemented and hence $G / N$ is supersolvable by induction. It follows that in order to prove that $G$ is supersolvable, it suffices to prove that $N=\langle x\rangle$ is cyclic. Let $P$ be a Sylow $p$-subgroup of $G$ and let $x \in N \cap Z(P)$ with $|x|=p$. Then, there exists $K \leq G$ such that $\langle x\rangle K=G$ and $\langle x\rangle \cap K$ is $S$-quasinormal in $K$. Since $\langle x\rangle \cap K$ is nomalized by all $p^{\prime}$-elements of $K$ and centralized by $P$, It follows that $\langle x\rangle \cap K$ is a normal subgroup of $G$. By minimality of $N,\langle x\rangle \cap K=1$ or $N \leq K$. Assume that $\langle x\rangle \cap K=1$. By order considerations, it follows that $N=\langle x\rangle$. Assume now that $N \leq K$. Then $\langle x\rangle=\langle x\rangle \cap K \leq N$ and so $N=\langle x\rangle$.

Let $P$ be a Sylow $p$-subgroup of $G$ and $H$ is a subgroup of $\Phi(P)$. Then by Lemma 2.4(4), $H$ is $S$-quasinormal in $G$. By Lemma 2.6, we deduce that $\Phi(P)$ is normal in $G$. Hence, $\Phi(P) \leq \Phi(G)$ and, therefore every Sylow subgroup of $G / \Phi(G)$ is elementary abelian. The last argument follows from Lemma 2.4(4).

$(2) \Rightarrow(3)$. This part follows from [15, Theorem 2].

$(3) \Rightarrow(1)$. Assume that every subgroup of $G / \Phi(G)$ is complemented and every subgroup of $\Phi(G)$ is $S$-quasinormal in $G$. Let $H$ be a subgroup of $G$. Then, there exists a subgroup $K / \Phi(G)$ of $G / \Phi(G)$ such that $(H \Phi(G) / \Phi(G))(K / \Phi(G))=G / \Phi(G)$ and $(H \Phi(G) / \Phi(G)) \cap(K / \Phi(G))=(H \cap K) \Phi(G) / \Phi(G)=1$. It follows that $H K=G$ and $H \cap K \leq \Phi(G)$. Hence, $H \cap K$ is $S$-quasinormal in $G$. By definition, $H$ is $S S$ supplemented in $G$ and hence $G$ is an $S S$-supplemented group. The proof of theorem is now complete. 
4. Applications. In this section, we concentrate on the structure of a group under the assumption that some subgroups of Sylow subgroups are $S S$-supplemented. Many known results will be generalized. In our first result, the $p$-nilpotency of a group is studied.

THEOREM 4.1. Let $G$ be a group and let $p$ be the smallest prime divisor of $|G|$. Let $P$ be a Sylow p-subgroup of $G$. If every maximal subgroup of $P$ is $S S$-supplemented in $G$, then $G$ is p-nilpotent.

Proof. Assume that the theorem is false and let $G$ be a counterexample of minimal order. Then, it follows from [16, IV, 2.8] that $P$ is not cyclic. Let $P_{1}$ be a maximal subgroup of $P$. Then, there exists $K \leq G$ such that $P_{1} K=G$ and $P_{1} \cap K$ is $S$-quasinormal in $K$. It follows from Lemma 2.6 and $\left|P \cap K: P_{1} \cap K\right| \leq p$ that $P_{1} \cap K$ is normal in $K$. Applying [16, IV, 2.8] again, $K / P_{1} \cap K$ is $p$-nilpotent with normal Hall $p^{\prime}$-subgroup $H / P_{1} \cap K$. Then, by the Schur-Zassenhaus theorem [9, Theorem 6.2.1], we know that $P_{1} \cap K$ has a $p$-complement $M$ in $H$. By using the Frattini argument, we deduce that $K=H N_{K}(M)=\left(P_{1} \cap K\right) N_{K}(M)$ and hence $G=P_{1} N_{G}(M)$. By the choice of $G$, it implies that $N_{G}(M)<G$ and $P \cap N_{G}(M)<P$. Now, choose a maximal subgroup $P_{2}$ of $P$ such that $P \cap N_{G}(M) \leq P_{2}$. By repeating the above argument once again, we can show that there also exists $K_{1} \leq G$ such that $P_{2} K_{1}=G$ and $P_{2} \cap K_{1}$ is $S$-quasinormal in $K_{1}$ and $G=P_{2} N_{G}\left(M_{1}\right)$, where $M_{1}$ is a Hall $p$-subgroup of $G$. If $p=2$, then by applying the Gross theorem [10, main theorem], we obtain that $M_{1}^{g}=M$ for some $g \in P$. If $p>2$, then the odd order theorem implies the same conclusion. Therefore, $G=P_{2} N_{G}\left(M_{1}\right)=\left(P_{2} N_{G}\left(M_{1}\right)\right)^{g}=P_{2} N_{G}(M)$. It follows that $P=P_{2}\left(P \cap N_{G}(M)\right)=P_{2}$, a contradiction. The proof is completed.

THEOREM 4.2. Let $\mathcal{F}$ be a saturated formation containing the class $\mathcal{U}$ of all supersoluble groups and $H$ a normal subgroup of a group $G$ such that $G / H \in \mathcal{F}$. If all maximal subgroups of every non-cyclic Sylow subgroup of $H$ are $S S$-supplemented in $G$, then $G \in \mathcal{F}$.

Proof. Let $p$ be the smallest prime divisor of $|H|$ and $P$ a Sylow $p$-subgroup of $H$. If $P$ is cyclic, then by [16, IV, 2.8], $H$ is $p$-nilpotent. If $P$ is non-cyclic, then by Lemma 2.4 (1) and Theorem 4.1, we deduce that $H$ is $p$-nilpotent. By using the same argument and induction, we may conclude that $H$ is a Sylow tower group.

Now, let $q$ be the largest prime dividing $|H|$ and $Q$ a Sylow $q$-subgroup of $H$. Then, $Q$ is normal in $G$. If $Q_{1}$ is a normal subgroup of $G$ with $1 \neq Q_{1} \leq Q$, then, by Lemma 2.4 (2) or (3), $G / Q_{1}$ satisfies the hypotheses of the theorem and therefore we have $G / Q_{1} \in \mathcal{F}$, by induction. If $Q_{1} \leq \Phi(G)$, then it follows from $G / Q_{1} \in \mathcal{F}$ that $G \in \mathcal{F}$. Hence, in this case, we may assume that $Q$ is not contained in $\Phi(G)$ and $Q$ is a minimal normal subgroup of $G$. If $Q$ is not a cyclic group, then we let $\left\{N_{1}, \ldots, N_{t}\right\}$ be the set of all maximal subgroups of $Q$. For each $N_{i}$, by the hypotheses, there exists $K_{i} \leq G$ such that $N_{i} K_{i}=G$ and $N_{i} \cap K_{i}$ is $S$-quasinormal in $K_{i}$. Hence, we have $Q=N_{i}\left(Q \cap K_{i}\right)$ and $Q \cap K_{i} \unlhd G$. By the minimality of $Q$, we deduce that $Q \cap K_{i}=1$ or $Q \leq K_{i}$. If $Q \cap K_{i}=1$, then $Q=N_{i}$, a contradiction. Thus, $Q \leq K_{i}$ and so $N_{i}$ is $S$-quasinormal in $G$. Now, Lemma 2.6 implies that $\left|G: N_{G}\left(N_{i}\right)\right|=q^{k}$ for some nonnegative integer $k$. It hence follows from [16, III, 8.5(d)] that some maximal subgroup of $N$ is normal in $G$, which is a contradiction. This shows that $Q$ is a cyclic group of order $q$. By Lemma 2.8, we conclude that $G \in \mathcal{F}$. The proof is completed.

The following corollary follows immediately from Theorem 4.2. 
Corollary 4.3. Let $N$ be a normal subgroup of a group $G$ such that $G / N$ is supersolvable. If every maximal subgroup of every Sylow subgroup of $N$ is c-supplemented in $G$, then $G$ is supersolvable.

THEOREM 4.4. Let $\mathcal{F}$ be a saturated formation containing the formation $\mathcal{U}$ of all supersoluble groups and $H$ a solvable normal subgroup of a group $G$ such that $G / H \in \mathcal{F}$. If all maximal subgroups of every Sylow subgroup of the Fitting subgroup $F(H)$ of $H$ are $S S$-supplemented in $G$, then $G \in \mathcal{F}$.

Proof. Let $M$ be a maximal subgroup of $G$ not containing $F(H)$. Then, by Lemma 2.9, it suffices to prove that $F(H) \cap M$ is maximal in $F(H)$. To proceed with the proof, let $P$ be a Sylow $p$-subgroup of $F(H)$ not contained in $M$ and let $G_{p}$ be a Sylow $p$-subgroup of $G$. Then, $P M=G$ and $G_{p} \cap M<G_{p}$. Choose a maximal subgroup $G_{1}$ of $G_{p}$ such that $G_{p} \cap M \leq G_{1}$ and let $P_{1}=G_{1} \cap P$. Then, $P_{1}$ is a maximal subgroup of $P$ and $P_{1} \cap M=P \cap M$. Now, we suppose that $P \cap \Phi(G) \neq 1$. Then, we can let $N$ be a minimal normal subgroup of $G$ contained in $P \cap \Phi(G)$. In this case, we have $F(H) / N=F(H / N)$ and $G / N$ satisfies the hypotheses. By using induction, we know that $G / N \in \mathcal{F}$ and therefore $G \in \mathcal{F}$. Hence, we may assume that $P \cap \Phi(G)=1$ and therefore $\Phi(P)=1$. Thus, $P \cap M \unlhd G$ and $P \cap M \leq\left(P_{1}\right)_{G}$. It hence follows that $\left(P_{1}\right)_{G} M<G$ and so $P \cap M=\left(P_{1}\right)_{G}$. By the hypotheses, there exists $K_{1} \leq G$ such that $P_{1} K_{1}=G$ and $P_{1} \cap K_{1}$ is $S$-quasinormal in $K_{1}$. If $Q$ is a Sylow $q$-subgroup of $K_{1}$ with $q \neq p$ then it is clear that $Q$ normalizes $P_{1} \cap K_{1}$. On the other hand, since $P K_{1}=G$ and $P$ is abelian, we have that $P \cap K_{1}$ is normal in $G$. It follows from $G_{p}=P G_{1}$ that $P_{1} \cap K_{1}=G_{1} \cap P \cap K_{1}$ is normalized by $G_{p}$. Therefore, we have $P_{1} \cap K_{1} \unlhd G$ and $P_{1} \cap K_{1} \leq\left(P_{1}\right)_{G}$. Let $K=K_{1}\left(P_{1}\right)_{G}$. Then, $P_{1} \cap K=\left(P_{1}\right)_{G}$. The maximality of $M$ implies that $(P \cap K) M=M$ or $(P \cap K) M=G$. If $(P \cap K) M=$ $M$, then $P \cap K \leq P \cap M=\left(P_{1}\right)_{G}$ and therefore $P \cap K=\left(P_{1}\right)_{G}=P_{1} \cap K$. It follows that $P_{1}=P$, a contradiction. Hence, $(P \cap K) M=G$. It follows that $P \cap K=P$ by order considerations and so $P \leq K$. This proves that $P_{1}=P_{1} \cap K=\left(P_{1}\right)_{G}=P \cap M$. Consequently, $|F(H): F(H) \cap M|=|P: P \cap M|=p$ and $F(H) \cap M$ is maximal in $F(H)$, as required.

COROLlARY 4.5. ([12]) Let $\mathcal{F}$ be a saturated formation containing $\mathcal{U}$. Let $H$ be a solvable normal subgroup of a group $G$ such that $G / H \in \mathcal{F}$. If all maximal subgroups of every Sylow subgroup of $F(H)$ are complemented in $G$, then $G \in \mathcal{F}$.

Now we want to delete the solvability of $H$ in the assumption of Theorem 4.4 by replacing $F(H)$ by $F^{*}(H)$, the generalized Fitting subgroup of $H$.

THEOREM 4.6. Let $G$ be a group with a normal subgroup $H$ such that $G / H$ is supersolvable. If every maximal subgroup of every Sylow subgroup of $F^{*}(H)$ is $S S$ supplemented in $G$, then $G$ is supersolvable.

Proof. Suppose that the theorem is false and let $G$ be a counterexample of minimal order. Then, every proper normal subgroup of $G$ containing $F^{*}(H)$ is supersolvable. In fact, let $N$ be a proper normal subgroup of $G$ containing $F^{*}(H)$. Then, $N / N \cap$ $H \cong N H / H$ is supersolvable. Since $F^{*}(H)=F^{*}\left(F^{*}(H)\right) \leq F^{*}(H \cap N) \leq F^{*}(H)$, we see $F^{*}(H \cap N)=F^{*}(H)$. Hence, every maximal subgroup of every Sylow subgroup of $F^{*}(H \cap N)$ is $S S$-supplemented in $G$ and therefore in $N$ by Lemma 2.4(1). So, $N$ with the normal subgroup $N \cap H$ satisfies the hypotheses of the theorem. The choice of $G$ implies that $N$ is supersolvable. 
If $H<G$, then $H$ is supersolvable. In this case, $F^{*}(H)=F(H)$. Theorem 4.4 implies that $G$ is supersolvable, a contradiction. Thus, $H=G$. If $F^{*}(G)=G$, then $G$ is supersolvable by Theorem 4.2 for the special case $\mathcal{F}=U$, a contradiction. Thus, $F^{*}(G)<G$. By the above proof, $F^{*}(G)$ is supersolvable and so $F^{*}(G)=F(G)$.

Let $P$ be a Sylow $p$-subgroup of $F(G)$. Suppose that $P \cap \Phi(G) \neq 1$, and let $N$ be a minimal normal subgroup of $G$ contained in $P \cap \Phi(G)$. Then, $F(G) / N=F(G / N)$ and $G / N$ satisfies the hypotheses. By the minimality of $G, G / N$ is supersolvable and so does $G$. Hence, $P \cap \Phi(G)=1$, and therefore $\Phi(P)=1$ and $P$ is abelian.

Let $P_{1}$ be a maximal subgroup of $P$. Then, there exists $K \leq G$ such that $P_{1} K=G$ and $P_{1} \cap K$ is $S$-quasinormal in $K$. Thus, $O^{p}(K) \leq N_{G}\left(P_{1} \cap K\right)$ and so $P_{1} \cap K \unlhd P O^{p}(K)$. Obviously, $F(G) \leq P O^{p}(K)$. Assume that $P O^{p}(K)<G$. Then, $P O^{p}(K)$ is supersolvable. Since $P O^{p}(K) \unlhd P K=G$ and $G / P O^{p}(K)$ is a $p$-group, $G$ is solvable. By Theorem 4.4, $G$ is supersolvable, a contradiction. Hence $P O^{p}(K)=$ $G$ and $P_{1} \cap K \unlhd G$. Therefore, $P_{1}$ is $C$-supplemented in $G$. Now applying [27, Theorem 1.1], we get $G$ is supersolvable, the final contradiction. The proof is hence completed.

THEOREM 4.7. Let $\mathcal{F}$ be a saturated formation containing the class $\mathcal{U}$ of all supersoluble groups and let $G$ be a group with a normal subgroup $H$ such that $G / H \in \mathcal{F}$. If every maximal subgroup of every Sylow subgroup of $F^{*}(H)$ is $S S$-supplemented in $G$, then $G \in \mathcal{F}$.

Proof. By Lemma 2.4(1), every maximal subgroup of every Sylow subgroup of $F^{*}(H)$ is $S S$-supplemented in $H$. Thus, $H$ is supersolvable by Theorem 4.6. In particular, $H$ is solvable and so $F^{*}(H)=F(H)$. Now Theorem 4.4 implies that $G \in \mathcal{F}$, as desired.

THEOREM 4.8. Let $G$ be a group and $p$ the smallest prime divisor of $|G|$. If every cyclic subgroup of $G$ with order $p$ and order 4 (if $p=2$ ) is $S S$-supplemented in $G$, then $G$ is p-nilpotent.

Proof. Assume that the theorem is false and let $G$ be a counterexample of minimal order. Then, by Lemma 2.4(1), $G$ is a minimal non- $p$-nilpotent group (that is, $G$ is not $p$-nilpotent but every proper subgroup of $G$ is $p$-nilpotent). Now by invoking a known result of Itô [16, III, 5.4], we know that $G$ is a minimal non-nilpotent group. According to a result of Schmidt in [16, III, 5.2], $G$ has a normal Sylow $p$-subgroup $P$ such that $G=P Q$ for a Sylow $q$-subgroup $Q(q \neq p)$.

Let $P_{0} \leq P$ with order $p$. Then, there exists $K \leq G$ such that $P_{0} K=G$ and $P_{0} \cap K$ is $S$-quasinormal in $K$. If $P_{0} \cap K=1$, then $K \unlhd G$ and $K$ is nilpotent. Thus, $Q \unlhd G$, which is a contradiction. If $P_{0} \leq K$, then $P_{0}$ is $S$-quasinormal in $G$ and therefore $P_{0} Q$ is a group. By the choice of $G$, we have $P_{0} Q<G$ and hence $P_{0} Q=P_{0} \times Q$. It follows that $Q$ centralizes $\Omega_{1}(P)$. If $C_{G}\left(\Omega_{1}(P)\right)<G$, then $C_{G}\left(\Omega_{1}(P)\right)$ is nilpotent and so $Q \unlhd G$, again a contradiction. This leads to $C_{G}\left(\Omega_{1}(P)\right)=G$ and $\Omega_{1}(P) \leq Z(G)$. If $\exp P=p$, then $G$ is $p$-nilpotent, a contradiction. Thus, $p=2$ and $\exp P=4$. Let $x \in P$ with $|\langle x\rangle|=4$. Then, there exists $T \leq G$ such that $\langle x\rangle T=G$ and $\langle x\rangle \cap T$ is $S$-quasinormal in $T$. If $|G: T|=4$, then $\left\langle x^{2}\right\rangle T \unlhd G$ and hence $Q \unlhd G$, again a contradiction. In the case $|G: T|=2$, we also have $Q \unlhd G$, the same contradiction. Therefore $T=G$ and $\langle x\rangle$ is $S$-quasinormal in $G$. By the choice of $G$, we have $\langle x\rangle Q<G$ and hence $\langle x\rangle$ centralizes $Q$. Thus, again we have $Q \unlhd G$, a contradiction. The proof is completed. 
Finally, we formulate another new theorem which also gives some other conditions for a finite group to be $p$-nilpotent.

THEOREM 4.9. Let $G$ be a group which is $A_{4}$-free and let $p$ be the smallest prime divisor of $|G|$. If every subgroup of $G$ having order $p^{2}$ is $S S$-supplemented in $G$, then $G$ is p-nilpotent.

Proof. Assume that the theorem is false and let $G$ be a counterexample of minimal order. Let $M$ be a maximal subgroup of $G$. Assume $|M|_{p} \leq p$. Then, by [16, IV, 2.8 ], $M$ is $p$-nilpotent. If $|M|_{p}>p$, then by Lemma 2.4 (1) and the choice of $G$ we can deduce that $M$ is $p$-nilpotent. Thus, $G$ is a minimal non- $p$-nilpotent group, and consequently, $G$ has a normal Sylow $p$-subgroup $P$ such that $G=P Q$, where $Q$ is a Sylow $q$-subgroup of $G$ with $q \neq p$.

Let $H \leq G$ with $|H|=p^{2}$. Then, there exists $K \leq G$ such that $H K=G$ and $H \cap$ $K$ is $S$-quasinormal in $K$. Without loss of generality, we may assume that $Q \leq K$. Suppose $H \cap K=1$, then $K$ is nilpotent. Let $K_{p}$ be a Sylow $p$-subgroup of $K$ and $P_{1}$ is a maximal subgroup of $P$ containing $K_{p}$. Then, $N_{K}\left(K_{p}\right)$ contains $P_{1}$ and $Q$. It follows that $\left|G: N_{K}\left(K_{p}\right)\right| \leq p$. If $\left|G: N_{K}\left(K_{p}\right)\right|=p$, then $N_{K}\left(K_{p}\right) \unlhd G$. However, it follows that $Q$ is normal in $G$, a contradiction. Assume that $K_{p} \unlhd G$. We consider the group $\bar{G}=G / K_{p}$. Clearly, $\bar{G} / C_{\bar{G}}(\bar{P})$ is isomorphic to a subgroup of $\operatorname{Aut}(\bar{P})$ so that $q \mid p^{2}-1=(p-1)(p+1)$. This implies that $p=2$ and $q=3$. Hence, $\bar{G} / \Phi(\bar{Q})$ is isomorphic to $A_{4}$, a contradiction.

If $|H \cap K|=p$, then $K \unlhd G$. Hence $Q \unlhd G$, again a contradiction.

Now, we have $H \leq K$ and thereby $H$ is $S$-quasinormal in $G$. If $H Q=G$, then $P=H$ is not cyclic. Clearly, $C_{G}(P)<G$. Now, $G / C_{G}(P)$ is isomorphic to a subgroup of $\operatorname{Aut}(P)$ so that $p=2$ and $q=3$. Hence, $G / \Phi(Q)$ is isomorphic to $A_{4}$, which is a contradiction. Thus, $H Q<G$ and $H Q$ is nilpotent. It follows that $P$ normalizes $Q$, which is a contradiction. Thus the proof is completed.

ACKNOWLEDGEMENTS. The authors would like to thank the referee for his or her valuable suggestions and useful comments contributed to the final version of this paper. The authors also would like to thank the referee for helping us to polish the final version of this paper. This research is partially supported by a grant of the National Natural Science Foundation of China (11071155) and Shanghai Leading Academic Discipline Project (J50101).

\section{REFERENCES}

1. M. Asaad and A. Shaalan, On supersolvablility of finite groups, Arch. Math. 53 (1989), $318-326$.

2. M. Asaad, On maximal subgroups of Sylow subgroups of finite groups, Comm. Algebra 26 (1998), 3647-3652.

3. R. Baer, Classes of finite groups and their properties, Illinois J. Math. 1 (1957), 115-187.

4. A. Ballester-Bolinches, J. Cossey and M. C. Pedraza-Aguilera, On products of finite supersoluble groups, Comm. Algebra 29 (2001), 3145-3152.

5. A. Ballester-Bolinches and X. Guo, On complemented subgroups of finite groups, Arch. Math. 72 (1999), 161-166.

6. A. Ballester-Bolinches and M. D. Pérez-Ramos, A question of R. Maier concerning formations, J. Algebra 182 (1996), 738-747.

7. A. Ballester-Bolinches, Y. Wang and X. Guo, C-supplemented subgroups of finite groups, Glasgow Math. J. 42 (2000), 383-389.

8. K. Doerk and T. Hawkes, Finite soluble groups (Walter de Gruyter, Berlin, 1992). 
9. D. Gorenstein, Finite groups (Harper \& Row, New York, 1968). 311-319.

10. F. Gross, Conjugacy of odd order Hall subgroups, Bull. London Math. Soc. 19 (1987),

11. X. Guo and K. P. Shum, The influence of minimal subgroups of focal subgroups on the structure of finite groups, J. Pure Appl. Algebra 169(1) (2002), 43-51.

12. X. Guo and K. P. Shum, Complementarity of subgroups and the structure of finite groups, Algebra Colloquium 13(1) (2006), 9-16.

13. X. Guo and K. P. Shum, Finite $p$-nilpotent groups with some subgroups c-supplemented, J. Aust. Math. Soc. 78(3) (2005), 429-439.

14. P. Hall, A characteristic property of soluble groups, J. London Math. Soc. 12 (1937), $198-200$.

15. P. Hall, Complemented groups, J. London Math. Soc. 12 (1937), 201-204.

16. B. Huppert, Endliche Gruppen I (Springer-Verlag, New York, Berlin, 1967).

17. N. Itô, Über das Produkt von zwei abelschen Gruppen, Math. Z. 62 (1955), 400-401.

18. O. H. Kegel, Produkte nilpotenter Gruppen, Arch. Math. 12 (1961), 90-93.

19. O. H. Kegel, Sylow-Gruppen and Subnormalteiler endlicher Gruppen, Math. Z. 78 (1962), 205-221.

20. V. M. Levchuk and A. G. Likharev, Finite simple groups with complement maximal sugroups, Siberian Math. J. 47(4) (2006), 659-668.

21. S. Li, Z. Shen, J. Liu and X. Liu, The influence of SS-quasinormality of some subgroups on the structure of finite groups, J. Algebra 319 (2008), 4275-4287.

22. S. Li, Z. Shen and X. Kong, On SS-quasinormal subgroups of finite groups, Comm. Algebra 36 (2008), 4436-4447.

23. R. Maier, A completeness property of certain formations, Bull. London Math. Soc. 24 (1992), 540-544.

24. P. Schmid, Subgroups permutable with all Sylow subgroups, J. Algebra 207 (1998), $285-293$.

25. A. N. Skiba, On weakly s-permutable subgroups of finite groups, J. Algebra 315 (2007), 192-209.

26. Y. Wang, H. Wei and Y. Li, A generalization of Kramer's theorem and its applications, Bull. Aus. Math. Soc. 65 (2002), 2193-2200.

27. H. Wei, Y. Wang and Y. Li, On $c$-supplemented maximal and minimal subgroups of Sylow subgroups of finite groups, Proc. Amer. Math. Soc. 132 (2004), 2197-2204.

28. H. Wielandt, Über den normalisator der subnormalen untergruppen, Math. Z. 69 (1958), 463-465.

29. H. Wielandt, Über Produkte von nilpotenten gruppen, Illinois J. Math. 2 (1958), 611618. 